\title{
ON THE $d$ TH POWER RESIDUE SYMBOL OF FUNCTION FIELDS
}

\author{
Su Hu
}

\begin{abstract}
In this short notice, we prove a new result about the $d$ th power residue symbol of function fields, by modifying the method of W. Kohnen in the paper published in Bull. Korean Math. Soc. 45 (2008), no. 2, 273-275.
\end{abstract}

W. Kohnen [1] gave a short and elementary proof of the existence of infinitely many primes $p$ such that a given positive integer $a$ congruent to 3 modulo 4 is a quadratic non-residue modulo $p$.

In this short notice, we prove a new result about the $d$ th power residue symbol of function fields by modifying the method of Kohnen [1]. Let $q$ be a power of an odd prime, $A=\mathbb{F}_{q}[t]$ be the polynomial ring over the finite field $\mathbb{F}_{q}$ with $q$ elements. Let $\mathbb{F}_{q}^{\times}$be the group of nonzero elements of $\mathbb{F}_{q}, g$ be a generator of $\mathbb{F}_{q}^{\times}$and $d$ be any divisor of $q-1$, thus $\eta=g^{\frac{q-1}{d}}$ becomes an element of order $d$ in $\mathbb{F}_{q}^{\times}$. For any irreducible polynomial $P$ in $A$, define $|P|=q^{\operatorname{deg} P}$. The $d$ th power residue symbol of $\mathbb{F}_{q}[t]$ is defined as follows [2].

Definition 1. Let $P \in A$ be an irreducible polynomial. $a \in A$ and $P$ does not divide $a$. Let $(a / P)_{d}$ be the unique element of $\mathbb{F}_{q}^{\times}$such that

$$
a^{\frac{|P|-1}{d}} \equiv\left(\frac{a}{P}\right)_{d} \quad(\bmod P) .
$$

If $P \mid a$ define $(a / P)_{d}=0$. The symbol $\left(\frac{a}{P}\right)_{d}$ is called the $d$ th power residue symbol.

Now we can state the following main result of this note.

Theorem 2. Let a be a nonzero polynomial in $\mathbb{F}_{q}[t]$ and $d \nmid \operatorname{deg} a$. Then for any $i=0,1,2, \ldots, d-1$, there exist infinitely many primes $P$ in $\mathbb{F}_{q}[t]$ with $d \nmid \operatorname{deg} P$, such that $\left(\frac{a}{P}\right)_{d}=\eta^{i \operatorname{deg} P}$.

When $d=2$, we have the following result.

Received September 13, 2008; Revised October 11, 2008.

2000 Mathematics Subject Classification. 11T55.

Key words and phrases. $d$ th power residue symbol, polynomial ring. 
Corollary 3. Let a be a nonzero polynomial in $\mathbb{F}_{q}[t]$ with odd degree. Then there exist infinitely many primes in $\mathbb{F}_{q}[t]$ with odd degree such that $\left(\frac{a}{P}\right)_{2}=-1$.

Now we modify Kohnen's method to give a short and elementary proof of Theorem 2.

Proof. For $x \in \mathbb{R}, x \geq q$, let

$$
m=-g^{i}\left(\prod_{|f| \leq x, a \neq 0(\bmod f)} f\right)^{d}+a^{\lambda},
$$

where in (1) the product over all primes $|f| \leq x$ that do not divide $a$ and $\lambda$ is a positive integer with $\lambda \equiv 1(\bmod d)$ such that

$$
\operatorname{deg} a^{\lambda}>\operatorname{deg}\left(\prod_{|f| \leq x, a \neq 0(\bmod f)} f\right)^{d} .
$$

Thus $\operatorname{deg} m=\lambda \operatorname{deg} a \equiv \operatorname{deg} a(\bmod d)$ and $d \nmid \operatorname{deg} m$.

Let $P$ be a prime dividing $m$ with $d \nmid \operatorname{deg} P$. Then necessarily, by the definition of $m$, we must have $|P|>x$.

On the other hand, we find from (1)

$$
a^{\lambda} \equiv g^{i}\left(\prod_{|f| \leq x, a \neq 0(\bmod f)} f\right)^{d}(\bmod P) .
$$

Thus

$$
a^{\lambda \frac{|P|-1}{d}} \equiv g^{i \frac{|P|-1}{d}}\left(\prod_{|f| \leq x, a \neq 0(\bmod f)} f\right)^{|P|-1}(\bmod P) .
$$

From Fermat's Little Theorem (see the corollary of Proposition 1.8 in [2]), we deduce that

$$
a^{\lambda \frac{|P|-1}{d}} \equiv g^{i \frac{|P|-1}{d}} \quad(\bmod P) .
$$

From the definition of the $d$ th power residue symbol of $\mathbb{F}_{q}[t]$, we get

$$
\left(\frac{a}{P}\right)_{d} \equiv a^{\frac{|P|-1}{d}} \equiv a^{\frac{|P|-1}{d} \lambda} \equiv g^{i \frac{|P|-1}{d}} \equiv \eta^{i \operatorname{deg} P} \quad(\bmod P) .
$$

Which conclude the proof.

\section{References}

[1] W. Kohnen, An elementary proof in the theory of quadratic residues, Bull. Korean Math. Soc. 45 (2008), no. 2, 273-275.

[2] M. Rosen, Number Theory in Function Fields, Springer-Verlag, New York, 2002.

Department of Mathematical Sciences

Tsinghua University

BeiJing 100084, P. R. China

E-mail address: hus04@mails.tsinghua.edu.cn 